\author{
УДК 621.923

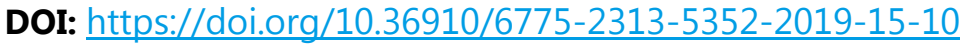 \\ Полянский В. И., К. т. н. \\ ООО «Империя металлов», г. Харьков
}

\title{
КОНЦЕПЦИИ ВЫСОКОТОЧНОЙ МЕХАНИЧЕСКОЙ ОБРАБОТКИ ОТВЕРСТИЙ В ДЕТАЛЯХ МАШИН
}

Теоретически определены условия повышения точности обработки отверстий при использовании мерных и немерных лезвийных инструментов и при шлифовании. Показано, что при обработке отверстий с неравномерно снимаемым припуском осевыми инструментами образуются упругие перемещения, снижающие точность обработки. Поэтому обработку отверстий в несколько проходов целесообразно осуществлять сверлами с разными диаметрами, увеличивая диаметр от прохода $\kappa$ проходу. Показано, что основным параметром, определяюшим погрешность обработки, является несоосность осевого инструмента и обрабатываемого отверстия. Теоретически установлено, что при обработке отверстий мерными инструментами, например, при растачивании, гораздо проще обеспечить требуемую точность, чем при обработке осевым инструментом. Для этого необходимо увеличивать скорость резания и уменьшать подачу $u$ глубину резания и применять высокоэффективные режущие инструменты из СТМ. При расфрезеровывании отверстия можно достичь еще большего повышения точности его обработки. Показано, что основным условием высокоточной обработки при внутреннем шилфовании является снижение энергоемкости процесса резания за счет уменьшения интенсивности трения в зоне резания.

Ключевые слова: рассверливание отверстия, растачивание, внутреннее шллифование, упругое перемещение, точность обработки, производительность обработки

Постановка проблемы в общем виде и ее связь с важнейшими научными и практическими задачами. Обеспечение высокоточной обработки отверстий в деталях машин является важным условием создания конкурентоспособной машиностроительной продукции. В успешном решении этой задачи важная роль принадлежит разработке эффективных технологических процессов финишной лезвийной и абразивной обработки отверстий. Наряду с повышением точности обработки, они должны обеспечивать и высокие показатели производительности и качества (бездефектность, уменьшение шероховатости обработанной поверхности и т.д.). В связи с этим возникает необходимость проведения теоретического анализа показателей точности лезвийной и абразивной обработки отверстий и выявления условия их повышения. Это позволит научно обоснованно подходить к разработке эффективных технологических процессов финишной лезвийной и абразивной обработки отверстий, обеспечивающих высокие показатели точности, качества и производительности обработки. В особой мере это относится к технологическим процессам рассверливания и расфрезеровывания отверстий, внутреннего шлифования. Исследования выполнены в соответствии с тематическим планом научно-практических работ ООО «Империя металлов» (г. Харьков).

Анализ последних достижений и публикаций. Вопросам обеспечения точности механической обработки отверстий в научно-технической литературе постоянно уделяется значительное внимание. В работах [1 - 4] рассмотрены общие подходы к определению условий повышения точности обработки отверстий лезвийными и абразивными инструментами. Показано, что вследствие неравномерности снимаемого припуска и возникающих упругих перемещений в технологической системе сложно при финишной обработке отверстий добиться требуемой точности. В работах [5 - 7] приведены теоретические решения по исправлению погрешности обработки отверстий для конкретных условий резания лезвийными инструментами и шлифования. Однако, для более полного представления о технологических возможностях повышения точности обработки отверстий необходимо располагать обобщенными теоретическими решениями, охватывающими все основные методы механической обработки отверстий. Для этого необходимо с единых позиций аналитически описать возникающие при абразивной и лезвийной обработке погрешности отверстий и на их основе определить пути их уменьшения.

Цель работы - теоретический анализ путей повышения точности обработки отверстий в деталях машин при их лезвийной обработке и при шлифовании. 
Материалы исследований. Традиционно финишную обработку отверстий производят мерными и немерными режущими инструментами. В последние годы получил также применение высокоэффективный метод расфрезеровывания отверстий на современных металлорежущих станках с ЧПУ типа «обрабатывающий центр», который позволяет существенно повысить точность и производительность обработки. Основным недостатком обработки мерными осевыми инструментами (сверлами, зенкерами, развертками) является превышение диаметра обработанного отверстия диаметра инструмента в связи с неравномерностью снимаемого припуска и возникающими упругими перемещениями в технологической системе, что, естественно, снижает точность обрабатываемого отверстия (точности его размера и формы). При обработке немерным инструментом (при растачивании отверстия резцом), наоборот, диаметр обработанного отверстия, как правило, меньше заданного диаметра вследствие возникающих в технологической системе упругих перемещений. Поэтому важно теоретически установить закономерности формирования погрешностей обработки в двух рассматриваемых случаях.

На рис. 1 представлена расчетная схема определения величины упругого перемещения (деформирования) сверла при рассверливании отверстия за один проход $\Delta_{1}$, когда оси сверла с центром $O$ и обрабатываемого отверстия с центром $O_{0}$ не совпадают на величину $A_{0}[6]$ :

$$
\Delta_{1}=\frac{2 \cdot \sigma \cdot S \cdot \cos \varphi \cdot \Delta_{0}}{K_{\text {рез }} \cdot c},
$$

где $\sigma$ - условное напряжение резания, $\mathrm{H} / \mathrm{m}^{2} ; K_{\text {рез }}=P_{z} / P_{y} ; P_{z}, P_{y}$ - тангенциальная и радиальная составляющие силы резания, действующие на лезвие сверла, $\mathrm{H} ; S$ - подача, м/об.; $c$ - приведенная жесткость сверла в радиальном направлении, Н/м; $\varphi$ - половина двойного угла в плане сверла; $\Delta_{0}-$ величина несоосности сверла и обрабатываемого отверстия, м.

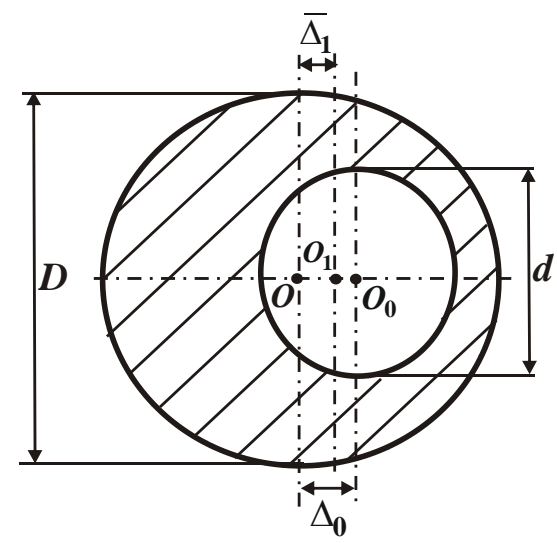

Рис. 1. Расчетная схема определения величины упругого перемещения сверла при рассверливании отверстия с начальным диаметром $d$

На втором проходе сверла величина его упругого деформирования $\Delta_{2}$ определяется этой же зависимостью (1), рассматривая в ней вместо величины $\Delta_{0}$ величину $\Delta_{1}$ :

$$
\Delta_{2}=\frac{2 \cdot \sigma \cdot S \cdot \cos \varphi \cdot \Delta_{1}}{K_{\text {рез }} \cdot c}=\left(\frac{2 \cdot \sigma \cdot S \cdot \cos \varphi}{K_{\text {рез }} \cdot c}\right)^{2} \cdot \Delta_{0} .
$$

Аналогично описывается величина упругой деформации сверла на третьем и на всех последующих $n$ проходах:

$$
\Delta_{3}=\left(\frac{2 \cdot \sigma \cdot S \cdot \cos \varphi}{K_{p e 3} \cdot c}\right)^{3} \cdot \Delta_{0}
$$




$$
\Delta_{n}=\left(\frac{2 \cdot \sigma \cdot S \cdot \cos \varphi}{K_{p e 3} \cdot c}\right)^{n} \cdot \Delta_{0}
$$

Отношение $\Delta_{0} / \Delta_{n}$ определяет суммарное уточнение $\varepsilon_{c y м}$ [2]:

$$
\varepsilon_{c y M}=\frac{\Delta_{0}}{\Delta_{n}}=\left(\frac{K_{p e s} \cdot c}{2 \cdot \sigma \cdot S \cdot \cos \varphi}\right)^{n} .
$$

Соответственно уточнение на проходе $\varepsilon$ равно:

$$
\varepsilon=\frac{\Delta_{0}}{\Delta_{1}}=\frac{\Delta_{1}}{\Delta_{2}}=\frac{\Delta_{2}}{\Delta_{3}}=\ldots=\frac{\Delta_{n-1}}{\Delta_{n}}=\frac{K_{\text {рез }} \cdot c}{2 \cdot \sigma \cdot S \cdot \cos \varphi} .
$$

Поскольку $\varepsilon>1$, то отношение $\frac{2 \cdot \sigma \cdot S \cdot \cos \varphi}{K_{p e s} \cdot c}<1$. Следовательно, с каждым последующим проходом сверла величина его упругой деформации будет уменьшаться $\Delta_{n} \rightarrow 0$, что обеспечит достижение заданной точности обрабатываемого отверстия.

Необходимо обратить внимание на то, что если обработка на первом проходе будет производиться сверлом с диаметром, равным заданному диаметру обрабатываемого отверстия, то это приведет к образованию погрешности обработки отверстия величиной $\Delta_{0}$. Причем, устранить эту погрешность на последующих проходах сверла невозможно. Поэтому обработку отверстий за несколько проходов целесообразно осуществлять сверлами с разными диаметрами, увеличивая диаметр от прохода к проходу. Аналогично устанавливаются диаметры зенкеров и разверток при обработке отверстий за несколько проходов, обеспечивая, таким образом, заданную точность обрабатываемого отверстия.

При растачивании отверстия резцом с неравномерно снимаемым припуском, т.е. при его обработке немерным инструментом, диаметр обрабатываемого отверстия формируется лишь за счет уменьшения величины упругого перемещения резца или детали, тогда как в предыдущем случае (при рассверливании отверстия) диаметр отверстия определялся суммой диаметра сверла и его упругого перемещения. Следовательно, при растачивании отверстия значительно проще добиться требуемой точности его обработки, чем при рассверливании. Для этого достаточно управлять лишь величиной упругого перемещения резца. Так, при первом проходе резца величина его упругого перемещения $y_{1}$ в радиальном направлении, исходя из соотношений [4]:

$$
\begin{gathered}
y_{1}=\frac{P_{y 1}}{c}=\frac{P_{z 1} \cdot \cos \varphi}{K_{p e 3} \cdot c}=\frac{\sigma \cdot S \cdot\left(t-y_{1}\right) \cdot \cos \varphi}{K_{p e 3} \cdot c}, \quad \text { равна: } \\
y_{1}=\frac{t}{\left(1+\frac{K_{p e 3} \cdot c}{\sigma \cdot S \cdot \cos \varphi}\right)},
\end{gathered}
$$

где $P_{y 1}, P_{z 1}$ - радиальная и тангенциальная составляющие силы резания при первом проходе, $\mathrm{H} ; c$ - приведенная жесткость технологической системы в радиальном направлении, $\mathrm{H} / \mathrm{m} ; \varphi$ главный угол резца в плане; $K_{p e з}=P_{z 1} / P_{y 1} ; S$ - подача, м/об.; $t$-глубина резания, м.

Уменьшить величину $y_{1}$ можно увеличением второго слагаемого, стоящего в знаменателе. При втором проходе сверла величина его упругого перемещения $y_{2}$ определяется: 


$$
y_{2}=\frac{\left(t+y_{1}\right)}{\left(1+\frac{K_{p e 3} \cdot c}{\sigma \cdot S \cdot \cos \varphi}\right)}=\frac{t}{\left(1+\frac{K_{p e 3} \cdot c}{\sigma \cdot S \cdot \cos \varphi}\right)}+\frac{t}{\left(1+\frac{K_{p e 3} \cdot c}{\sigma \cdot S \cdot \cos \varphi}\right)^{2}} .
$$

При $n$-ом проходе имеем:

$$
y_{n}=\frac{t}{\left(1+\frac{K_{p e 3} \cdot c}{\sigma \cdot S \cdot \cos \varphi}\right)}+\frac{t}{\left(1+\frac{K_{p e 3} \cdot c}{\sigma \cdot S \cdot \cos \varphi}\right)^{2}}+\ldots+\frac{t}{\left(1+\frac{K_{p e 3} \cdot c}{\sigma \cdot S \cdot \cos \varphi}\right)^{n}} .
$$

Характер изменения величины $y_{n}$ подчиняется геометрической прогрессии, тогда [8]:

$$
y_{n}=y_{1} \cdot \frac{\left(1-\varepsilon^{-n}\right)}{(1-\varepsilon)}=\frac{\sigma \cdot S \cdot t \cdot \cos \varphi}{K_{p е з} \cdot c} \cdot\left(1-\varepsilon^{-n}\right)=\frac{\sigma \cdot Q \cdot \cos \varphi}{K_{p e 3} \cdot c \cdot V} \cdot\left(1-\varepsilon^{-n}\right),
$$

где $\varepsilon=1+\frac{K_{p e з} \cdot c}{\sigma \cdot S \cdot \cos \varphi}-$ уточнение на проходе; $Q=S \cdot t \cdot V-$ производительность обработки, $\mathrm{m}^{3} / \mathrm{c}$.

Исходя из зависимости (10) уменьшить величину $y_{n}$ при заданной производительности обработки $Q=S \cdot t \cdot V$ можно путем увеличения скорости резания $V$, пропорционально уменьшая подачу $S$ или глубину резания $t$. При этом с уменьшением подачи $S$ увеличивается уточнение на проходе $\varepsilon$, уменьшается величина $\varepsilon^{-n}$ и увеличивается множитель $\left(1-\varepsilon^{-n}\right)$, что не позволяет пропорционально уменьшать величину $y_{n}$ с увеличением скорости резания $V$.

Уменьшение глубины резания $t$ при заданном значении снимаемого припуска П приводит к увеличению $n=\Pi / t$ и соответственно к уменьшению величина $\varepsilon^{-n}$ и увеличению множителя $\left(1-\varepsilon^{-n}\right)$, а это приводит к увеличению величины $y_{n}$. Поэтому с увеличением скорости резания $V$ (при пропорциональном уменьшении глубины резания $t$ и выполнении условия $Q=$ const ) величина $y_{n}$ будет уменьшаться, однако не по линейному закону в связи с увеличением множителя $\left(1-\varepsilon^{-n}\right)$ в зависимости (10).

Более эффективным решением, вытекающим из зависимости (10), следует рассматривать возможность увеличения производительности обработки $Q=S \cdot t \cdot V$ за счет увеличения скорости резания $V$. В этом случае будет выполняться условие $y_{n}=$ const при неизменных значениях подачи $S$ и глубины резания $t$ и увеличивающемся значении скорости продольной подачи $S_{n р о д ~}$, определяемой из условия: $\tau=\frac{S}{S_{n р о д}}=\frac{\pi \cdot D_{\text {дет }}}{V}$, где $\tau-$ время перемещения резца в продольном направлении на величину подачи $S$, с; $D_{\text {дет }}$ - диаметр детали, м. Тогда

$$
S_{\text {прод }}=\frac{S \cdot V}{\pi \cdot D_{\text {дет }}} .
$$

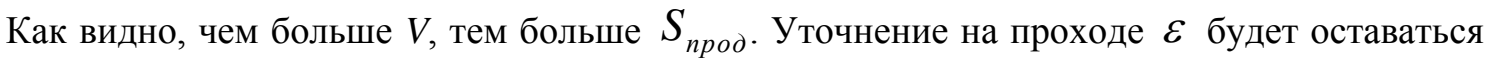
неизменным, поскольку подача $S$ в этом случае является неизменной.

Для обеспечения заданной точности обработки, исходя из зависимости (10), необходимо уменьшать отношение $\sigma / K_{\text {рез }}$ и увеличивать жесткость технологической системы $c$. При этом уточнение на проходе $\varepsilon$ увеличивается, что приводит к уменьшению величины $\varepsilon^{-n}$ и увеличению множителя $\left(1-\varepsilon^{-n}\right)$, однако не столь существенно по сравнению с уменьшением $y_{n}$ за счет линейного уменьшения отношения $\sigma / K_{\text {рез }}$ и увеличения жесткости 
технологической системы $c$. В итоге величину $y_{n}$ можно уменьшить до необходимого значения.

Полученные теоретические решения согласуются с практикой растачивания отверстий, где на финишных операциях, например, с применением резцов из синтетических сверхтвердых материалов (СТM) обработку осуществляют с увеличенной скоростью резания $V$ и относительно небольшими значениями подачи $S$ или глубины резания $t$. Это обеспечивает повышение точности и производительности обработки. Кроме того, применение резцов из синтетических сверхтвердых материалов (СТM), обладающих высокой остротой и режущей способностью, позволяет уменьшить отношение $\sigma / K_{\text {рез }}$, что дополнительно снижает величину $y_{n}$ и повышает точность обработки.

Используя зависимость (10), произведем анализ возможностей уменьшения величины $y_{n}$ при внутреннем шлифовании. В этом случае угол $\varphi=0 ; \cos \varphi=1 ; \quad V=V_{\text {дет и, }}$ и, соответственно, все закономерности, установленные для лезвийной обработки, остаются справедливыми и для внутреннего шлифования. Поэтому целесообразно увеличивать скорость детали $V_{\text {дет }}$ при одновременном уменьшении подачи $S$ и глубины шлифования $t$. Это способствует повышению точности и производительности обработки. Важно также обеспечить уменьшение отношения $\sigma / K_{\text {рез }}$ и увеличение жесткости технологической системы $c$.

Как известно, при шлифовании параметр $\sigma$ больше, чем при лезвийной обработке из-за наличия интенсивного трения связки шлифовального круга с обрабатываемым материалом. Поэтому уточнение на проходе $\varepsilon$ будет меньше, что приведет к увеличению величины $\varepsilon^{-n}$ и уменьшению множителя $\left(1-\varepsilon^{-n}\right)$ в зависимости (10). Это в определенной степени будет компенсировать увеличение величины $y_{n}$ с увеличением параметра $\sigma$. Исходя из сказанного, увеличение величины $y_{n}$ при внутреннем шлифовании не будет столь значительным по сравнению с лезвийной обработкой. При глубинном внутреннем шлифовании с относительно небольшой скоростью детали $V_{\text {дет }}$ величина $y_{n}$ увеличится. Следовательно, целесообразно использовать схему многопроходного внутреннего шлифования.

При расфрезеровывании отверстия величина $y_{n}$ подчиняется тем же закономерностям, что и при растачивании и внутреннем шлифовании [9]. Следовательно, эффективно увеличивать скорость резания, т.е. обработку производить в условиях высокоскоростного резания. Благодаря увеличению количества одновременно работающих режущих лезвий фрезы, это позволит повысить производительность и точность обработки по сравнению с процессами растачивания и внутреннего шлифования и, тем более, по сравнению с процессом рассверливания отверстия. Таким образом показано, что с точки зрения повышения производительности и точности методы механической обработки отверстий немерными инструментами (резцами, шлифовальными кругами, фрезами) располагают большими технологическими возможностями, чем методы обработки мерными осевыми инструментами (сверлами, зенкерами, развертками).

Выводы. Теоретически определены условия повышения точности обработки отверстий при использовании мерных и немерных лезвийных инструментов и при шлифовании. Показано, что при обработке отверстий с неравномерно снимаемым припуском осевыми инструментами образуются упругие перемещения, снижающие точность обработки. Поэтому обработку отверстий за несколько проходов целесообразно осуществлять сверлами с разными диаметрами, увеличивая диаметр от прохода к проходу. Установлено, что при обработке отверстий мерными инструментами, например, при растачивании и расфрезеровывании, гораздо проще обеспечить требуемую точность, чем при рассверливании. Показано, что основным условием высокоточной обработки при внутреннем шлифовании является снижение энергоемкости процесса резания за счет уменьшения интенсивности трения в зоне резания.

Перспективы дальнейшей работы в данном направлении. В дальнейших исследованиях необходимо теоретически и экспериментально определить условия уменьшения 
энергоемкости процесса резания, оказывающего значительное влияние на точность обрабатываемого отверстия при его рассверливании, растачивании и внутреннем шлифовании.

\section{Информационные источники}

1. Бобров В. Ф. Основы теории резания металлов. - М.: Машиностроение, 1975. - 343 с.

2. Маталин А. А. Технология машиностроения: учебник. - Л.: Машиностроение, 1985. - 496 c.

3. Сизый Ю. А., Сталинский Д. В. Динамика и теплофизика шлифования. - Харьков: ГП УкрНТЦ «Энергосталь», 2016. - 448 с.

4. Физико-математическая теория процессов обработки материалов и технологии машиностроения / Под общ. ред. Ф. В. Новикова и А. В. Якимова. В десяти томах. - Т. 1. «Механика резания материалов». - Одесса: ОНПУ, 2002. - 580 с.

5. Лурье Г. Б. Прогрессивные методы круглого наружного шлифования. - Л.: Машиностроение, 1984. - 103 с.

6. Иванов И. Е. Технологическое обеспечение точности и стабильности изготовления резьбовых отверстий в баллонах в условиях массового производства: автореф. дис. ... канд. техн. наук: специальность 05.02.08 «Технология машиностроения». - Мариуполь, 2008. - 21 с.

7. Новоселов Ю. К. Динамика формообразования поверхностей при абразивной обработке. - Саратов, 1979. - $232 \mathrm{c}$.

8. Теоретичні основи механічної обробки високоточних деталей: монографія / Ф. В. Новіков, І. О. Рябенков. - Х. : Вид. ХНЕУ, 2013. - 352 с.

9. Полянский В. И. Расширение технологических возможностей повышения точности механической обработки отверстий // Перспективні технології та прилади: Збірник наукових праць. - Луцьк: Луцький НТУ, 2017. - №11 (2). - С. 87-92.

Полянський В. І., к.Т.н.

ТОВ «Імперія металів», м. Харків

\section{КОНЦЕПЦІЇ ВИСОКОТОЧНОЇ МЕХАНІЧНОЇ ОБРОБКИ ОТВОРІВ В ДЕТАЛЯХ МАШИН}

Теоретично визначено умови підвищення точності обробки отворів при використанні мірних і немірних лезових інструментів і при шліфуванні. Показано, щзо при обробиі отворів з нерівномірно знімаємим припуском осьовими інструментами утворюються пружні переміщення, що знижують точність обробки. Тому обробку отворів в кілька проходів доцільно здійснювати свердлами з різними діаметрами, збільшуючи діаметр від проходу до проходу. Показано, щчо основним параметром, що визначає похибку обробки, є неспіввісність осьового інструменту $і$ оброблюваного отвору. Теоретично встановлено, щзо при обробиі отворів мірними інструментами, наприклад, при розточуванні, набагато простіше забезпечити необхідну точність, ніж при обробиі осьовим інструментом. Для цього необхідно збільшувати швидкість різання $і$ зменшувати подачу $i$ глибину різання та застосовувати високоефективні різальні інструменти з СНМ. При розфрезеруванні отвору можна досягти ще більшого підвищення точності його обробки. Показано, що основною умовою високоточної обробки при внутрішньому шліфуванні є зниження енергоємності прочесу різання за рахунок зменшення інтенсивності тертя в зоні різання.

Ключові слова: розсвердлювання отвору, розточування, внутрішне шліфування, пружне переміщення, точність обробки, продуктивність обробки

Polyansky V. I., Ph.D.

LLC "Empire of metals", Kharkiv

\section{CONCEPTS OF HIGH-PRECISION MECHANICAL MACHINING OF OPENINGS IN MACHINE PARTS}

Theoretically, the conditions for increasing the accuracy of hole machining when using dimensional and non-dimensional blade tools and during grinding are theoretically determined. It is shown that when machining holes with uneven allowance with axial tools, elastic displacements are formed that reduce the accuracy of the machining. Therefore, it is advisable to process holes in several passes with drills with different diameters, increasing the diameter from the passage to the 
passage. It is shown that the main parameter determining the processing error is the misalignment of the axial tool and the machined hole. It has been theoretically established that when machining holes with measuring tools, for example, during boring, it is much easier to provide the required accuracy than when machining with an axial tool. To do this, it is necessary to increase the cutting speed and reduce the feed and depth of cut and apply high-performance cutting tools from STM. When milling holes, you can achieve even greater increase in the accuracy of its processing. It is shown that the main condition for high-precision machining during internal grinding is to reduce the energy intensity of the cutting process by reducing the friction intensity in the cutting zone.

Key words: hole drilling, boring, internal grinding, elastic displacement, machining accuracy, machining productivity 